\title{
Low-Grazing Angle Detection in Compound-Gaussian Clutter with Hybrid MIMO Radar
}

\author{
Jincan Ding, Haowen Chen, Hongqiang Wang, Xiang Li, and Zhaowen Zhuang \\ College Electronic Science and Engineering, National University of Defense Technology, Changsha 410073, China \\ Correspondence should be addressed to Jincan Ding; jincanding@163.com
}

Received 15 April 2013; Revised 12 September 2013; Accepted 17 September 2013

Academic Editor: Hang Hu

Copyright ( 2013 Jincan Ding et al. This is an open access article distributed under the Creative Commons Attribution License, which permits unrestricted use, distribution, and reproduction in any medium, provided the original work is properly cited.

\begin{abstract}
This paper focuses on the target detection in low-grazing angle using a hybrid multiple-input multiple-output (MIMO) radar systems in compound-Gaussian clutter, where the multipath effects are very abundant. The performance of detection can be improved via utilizing the multipath echoes. First, the reflection coefficient considering the curved earth effect is derived. Then, the general signal model for MIMO radar is introduced in low-grazing angle; also, the generalized likelihood test (GLRT) and generalized likelihood ratio test-linear quadratic (GLRT-LQ) are derived with known covariance matrix. Via the numerical examples, it is shown that the derived GLRT-LQ detector outperforms the GLRT detector in low-grazing angle, and both performances can be enhanced markedly when the multipath effects are considered.
\end{abstract}

\section{Introduction}

MIMO radar has gotten considerable attention in a novel class of radar system, where the term MIMO refers to the use of multiple-transmit as well as multiple-receive antennas. MIMO radar is categorized into two classes: the statistical MIMO radar and the colocated MIMO radar, depending on their antenna placement $[1,2]$. The advantages of MIMO radar with colocated antennas have been studied extensively, which include improved detection performance, higher resolution [3], higher sensitivity to or detection of moving targets [4], and increased degrees of freedom for transmission beamforming [5]. MIMO radar with widely separated antennas can capture the spatial diversity of the target's radar cross section (RCS) [6]. This spatial diversity provides the radar systems with the ability to support the improvement of the target parameter estimation $[7,8]$, high resolution target localization [9], and tracking performance [10]. The hybrid MIMO radars can obtain superiority both from colocated and separated MIMO radar. Thus, we focus on the hybrid MIMO radar system in this paper.

Much published literature has concerned the issue of MIMO radar detection. Guan and Huang [11] investigated the detection problem of the MIMO radar system with distributed apertures in Gaussian colored noise and partially correlated observation channels. Tang et al. [12] introduced relative entropy as a measure to radar detection theory and analyzed the detection performance of MIMO radar and phased array radar. The authors in [13] investigated detection performance of MIMO radar for Rician target. In [14], the optimal detector in the Neyman-Pearson sense was derived for the statistical MIMO radar using orthogonal waveforms. The authors in [15] applied the Swerling models to target detection and derived the optimal test statistics for a statistical MIMO radar using nonorthogonal signal. For low-grazing angle detection of MIMO radar, the authors in [16] utilized the time reversal technique in a multipath environment to achieve high target detectability.

Low-grazing angle targets are difficult to detect, which is one of the great threats propelling radar development. Otherwise, detection of low-altitude targets is of great significance to counter low-altitude air defense penetration. However, up to now, this problem has not been effectively resolved. Multipath effect plays an important role in the lowaltitude target detection, by which the target echo signal is seriously polluted, even counteracted [17]. Two aspects can be considered for multipath: suppressing multipath and utilizing it. However, in a statistical sense, detection may be enhanced by the presence of multipath [18]. 
In this paper, we consider low-grazing angle target detection in compound-Gaussian clutter for MIMO radar. The compound-Gaussian clutter represents the heavy-tailed clutter statistics that are distinctive of several scenarios, for example, high-resolution or low-grazing angle radars in the presence of sea or foliage clutter $[19,20]$. To the end, the generalized likelihood ratio test (GLRT) and generalized likelihood ratio test-linear quadratic (GLRT-LQ) are derived.

\section{Multipath Geometry Model}

A point source at a distance of $R_{d}$ from the receiver is considered. If the source is assumed to be a narrowband signal, it can be represented by

$$
x(t)=a e^{j(\omega t+\varphi)},
$$

where $a$ is the amplitude, $\omega$ is the angular frequency, and $\varphi$ is the initial phase. In the presence of multipath, the received by the receiver consists of two components, namely, the direct and indirect signal. For a simple multipath model of a flat earth, the direct signal is given by

$$
x_{d}(t)=x(t) e^{-j \kappa R_{d}},
$$

while indirect signal is

$$
x_{i}(t)=x(t) \rho e^{j \phi} e^{-j \kappa R_{i}},
$$

where $\rho e^{j \phi}$ is the complex reflection coefficient, $\kappa=2 \pi / \lambda$ is the wave number, $\lambda$ is wavelength, target range $R_{d}$ can be obtained from the time delay, and $R_{i}$ is the total length of the indirect path. Thus, the total received signal is given by

$$
x_{r}(t)=x_{d}(t)+x_{i}(t) .
$$

To model the received signals more accurately, the curvature of the signal path due to refraction in the troposphere, in addition to the curvature of the earth itself, must be taken into account. The multipath geometry for a curved earth is given in Figure 1.

In (3), the term $\rho e^{j \phi}$ is the complex reflection coefficient. It generally consists of the Fresnel reflection coefficient divided into the vertical polarization $\Gamma_{v}$ and horizontal polarization $\Gamma_{h}$, the divergence factor $D$ due to a curved surface, and the surface roughness factor; that is, $\rho e^{j \phi}=\Gamma_{(v, h)} D \rho_{s}$. The vertical polarization and horizontal polarization Fresnel reflection coefficients are, respectively, as presented in [17]. Consider the following:

$$
\begin{gathered}
\Gamma_{v} \simeq \frac{\psi \sqrt{\varepsilon_{c}}-1}{\psi \sqrt{\varepsilon_{c}}+1}, \\
\Gamma_{h} \simeq \frac{\psi-\sqrt{\varepsilon_{c}}}{\psi+\sqrt{\varepsilon_{c}}} .
\end{gathered}
$$

For horizontal polarization, $\psi$ is the grazing angle and $\varepsilon_{c}$ is the complex dielectric constant which is given by

$$
\varepsilon_{c}=\frac{\varepsilon}{\varepsilon_{0}}-j 60 \lambda \sigma
$$

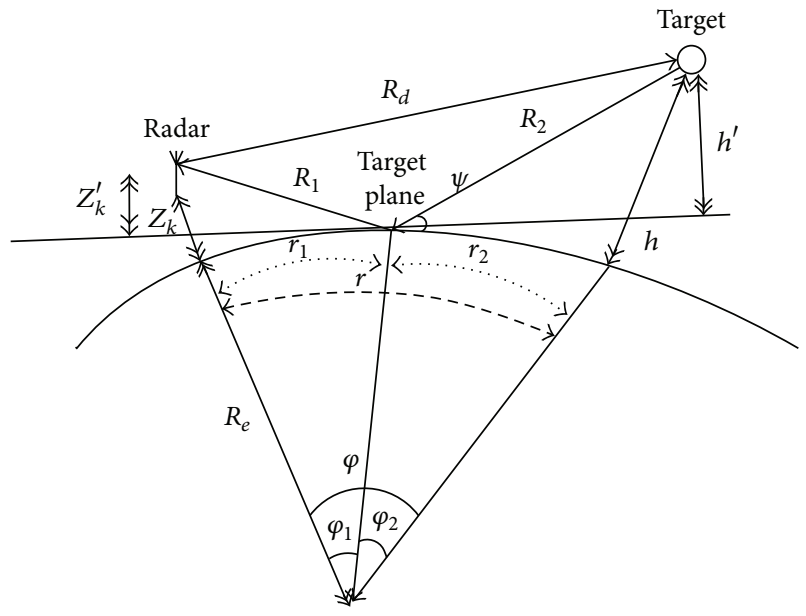

FIGURE 1: Multipath geometry for a curved earth.

where $\varepsilon / \varepsilon_{0}$ is the relative dielectric constant of the reflecting medium and $\sigma$ is its conductivity. Thus, the Fresnel reflection coefficient is determined by the grazing angle under a deterministic condition.

When an electromagnetic wave is incident on a round earth surface, the reflected wave diverges because of the earth's curvature. Due to divergence, the reflected energy is defocused and radar power density is reduced. The divergence factor can be derived solely from geometrical considerations. A widely accepted approximation for the divergence factor $D$ is given by

$$
D \simeq\left(1+\frac{2 r_{1} r_{2}}{R_{e} r \psi}\right)^{-1 / 2} .
$$

The surface roughness factor $\rho_{s}$ is given by

$$
\begin{gathered}
\rho_{s}=e^{-\mu}, \\
\mu= \begin{cases}2[2 \pi \eta]^{2}, & \eta \leq 0.1 \mathrm{rad}, \\
0.16 \eta^{2}+7.42 \eta+0.0468, & \text { otherwise }\end{cases}
\end{gathered}
$$

and $\eta$ is the surface roughness factor given by

$$
\eta=\frac{\sigma_{H} \psi}{\lambda}
$$

and $\sigma_{H}$ is the root-mean-square (RMS) surface height irregularity. For simplicity, the diffuse component is treated as the incoherent white Gaussian noise.

\section{MIMO Radar Multipath Signal}

Consider a narrowband MIMO radar system with $\widetilde{M}$ and $\widetilde{N}$ subarrays for transmitting and receiving, respectively. The $m$ th transmit and $n$th receive subarrays have, respectively, $M_{m}$ and $N_{n}$ closely spaced antennas. $m=1, \ldots, \widetilde{M}$, and $n=1, \ldots, \widetilde{N}, M=M_{1}+M_{2}+\cdots+M_{\widetilde{M}}$ and $N=N_{1}+$ $N_{2}+\cdots+N_{\widetilde{N}}$ are the total numbers of transmit and receive antennas, respectively. We assume that the subarrays are 


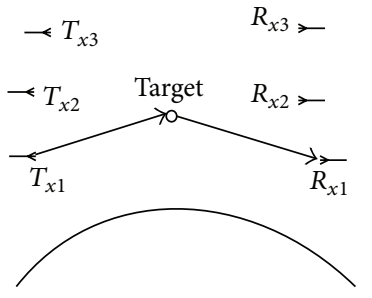

(a) Directly-directly path

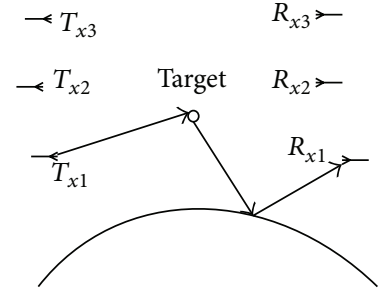

(b) Directly-reflected path

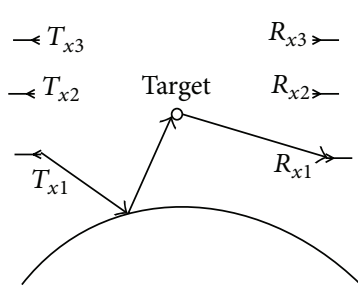

(c) Reflected-directly path

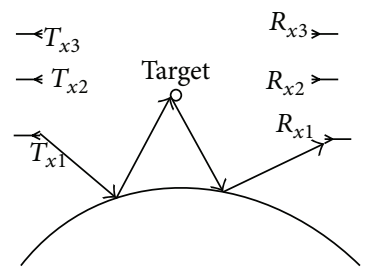

(d) Reflected-reflected path

FIGURE 2: Multipath MIMO radar.

sufficiently separated, and, hence, for each target, its RCSs for different transmit and receive subarray pairs are statistically independent of each other. The receive signal of MIMO radar can be expressed as [21]

$$
\mathbf{Y}=\mathbf{A}(\phi) \mathbf{B}_{\phi} \mathbf{S}(\phi)+\mathbf{Z}
$$

where $\mathbf{A}(\phi)=\operatorname{diag}\left\{\mathbf{a}_{1}(\phi), \mathbf{a}_{2}(\phi), \ldots, \mathbf{a}_{\widetilde{N}}(\phi)\right\}$ is the steering matrix of receive subarrays and component $\left\{\mathbf{a}_{n}(\phi)\right\}_{n=1}^{\widetilde{N}}$ is the steering vector of $n$th receive subarray at direction $\phi . \mathbf{B}_{\phi}$ denotes the RCSs for different transmit and receive subarray pairs with component $\left\{\beta_{m n, \phi}\right\}_{m, n=1,1}^{\widetilde{M}, \widetilde{N}} . \mathbf{S}(\phi)=\mathbf{V}^{T}(\phi) \Phi$ denotes the transmit signal matrix, where $\boldsymbol{\Phi}=\left[\boldsymbol{\Phi}_{1}^{T}, \boldsymbol{\Phi}_{2}^{T}, \ldots, \boldsymbol{\Phi}_{\widetilde{M}}^{T}\right]^{T}$ is the transmit waveform matrix, for each transmit subarray, the component $\boldsymbol{\Phi}_{m}^{\widetilde{M} \times L}=\left[\left\{s_{m 1}(t)\right\}^{T},\left\{s_{m 2}(t)\right\}^{T}, \ldots,\left\{s_{m M_{m}}(t)\right\}^{T}\right]^{T}$, and $\left\{s_{m l}(t)\right\}_{\ell=1}^{M_{m}}, t=1,2, \ldots, L$ is the probing waveform of $m$ subarray; $\mathbf{V}(\phi)=\operatorname{diag}\left\{\mathbf{v}_{1}(\phi), \mathbf{v}_{2}(\phi), \ldots, \mathbf{v}_{\widetilde{M}}(\phi)\right\}$ is the steering matrix of transmit subarray and the component $\left\{\mathbf{v}_{m}(\phi)\right\}_{m=1}^{\widetilde{M}}$ is the steering vector of $m$ th transmit subarray at direction $\phi . \mathbf{Y}=\left[Y_{1}^{T}, Y_{2}^{T}, \ldots, Y_{\widetilde{N}}^{T}\right]$ is the received data matrix and $\left\{Y_{n}\right\}_{n=1}^{\widetilde{N}}$ denotes received signal of the $n$th subarray. $\mathbf{Z}=\left[\mathbf{z}_{1}, \mathbf{z}_{2}, \ldots, \mathbf{z}_{L}\right]$ is the clutter matrix, each column $\left\{\mathbf{z}_{\ell}\right\}_{\ell=1}^{L}$ of which is modeled as spherically invariant random vectors (SIRV), and $L$ is the number of data samples of the transmitted waveforms. We assume clutter distributing as the compound-Gaussian model, which represents the heavytailed clutter statistics that are distinctive of several scenarios, for example, high-resolution or low-grazing angle radars in the presence of sea or foliage clutter $[19,20]$. The compoundGaussian clutter $z=\sqrt{u} \chi$, where $u$ and $\chi$ are the texture and speckle components of the compound model, respectively. The fast-changing $\chi$ is a realization of a stationary zero mean complex Gaussian process, and the slow-changing $u$ is modeled as a nonnegative real random process [22].

We rewrite the received signal (11) in vector form, given by

$$
\mathbf{y}_{\mathrm{dd}}=\mathbf{T} \boldsymbol{\beta}+\mathbf{n}
$$

where $\mathbf{y}^{N L \times 1}=\operatorname{Vec}(\mathbf{Y}), \mathbf{T}^{N L \times \widetilde{M} \widetilde{N}}=\left(\mathbf{S}^{T} \otimes \mathbf{A}\right), \boldsymbol{\beta}^{\widetilde{M} \widetilde{N} \times 1}=$ $\operatorname{Vec}\left(\mathbf{B}_{\phi}\right), \mathbf{n}^{N L \times 1}=\operatorname{Vec}(\mathbf{Z}), \operatorname{Vec}(\bullet)$ is the vector operator, symbol $\otimes$ denotes the Kronecker product. Then and $\mathbf{n}$ is the compound Gaussian random vector with covariance matrix

$$
\begin{gathered}
\mathbf{C}_{\mathbf{n}}^{N L \times N L}=E\left[\mathbf{n n}^{\dagger}\right]=\mathbf{P} \otimes \mathbf{C}, \\
\mathbf{P}=\operatorname{diag}\left\{s_{1}^{2}, s_{2}^{2}, \ldots, s_{L}^{2}\right\}=\operatorname{diag}\left\{\lambda_{1}, \lambda_{2}, \ldots, \lambda_{L}\right\},
\end{gathered}
$$

where $\mathbf{P}$ is considered deterministic matrix with unknown parameters $\left\{\lambda_{\ell}\right\}_{\ell=1}^{L}$ and $\dagger$ denotes conjugate transpose.

\section{Multipath Signal Model of MIMO Radar}

In the presence of multipath, consider atmosphere refraction and the curved earth effect; the reflected signals from a point target of MIMO radar include four parts: directlydirectly path, directly-reflected path, and reflected-directly path, reflected-reflected path. Assume the point target is located at $X_{0}=\left(x_{0}, y_{0}\right)$ and reflected point in ground is located at $X_{i}=\left(x_{i}, y_{i}\right), i=1,2$. Figure 2 illustrates a fourway MIMO radar propagation model with multipath.

The directly-directly path echo signal is given by (12). The directly-reflected path echo signal is

$$
\mathbf{y}_{\mathrm{dr}}=\mathbf{T}\left(\mathbf{k}^{\mathrm{dr}} \odot \boldsymbol{\beta}\right)+\mathbf{n},
$$

where $\mathbf{K}^{\mathrm{dr}}$ is $\widetilde{M} \widetilde{N} \times 1$ amplitude of reflect coefficient and symbol $\odot$ represents the Hadamard product.

The reflected-directly path echo signal is

$$
\mathbf{y}_{\mathrm{rd}}=\mathbf{T}\left(\mathbf{k}^{\mathrm{rd}} \odot \boldsymbol{\beta}\right)+\mathbf{n} \text {. }
$$

The reflected-reflected path echo signal is

$$
\mathbf{y}_{\mathrm{rr}}=\mathbf{T}\left(\mathbf{k}^{\mathrm{rr}} \odot \boldsymbol{\beta}\right)+\mathbf{n} .
$$
is

Thus, the received signal of MIMO radar with multipath

$$
\begin{aligned}
\mathbf{y}_{\mathrm{mp}} & =\mathbf{y}_{\mathrm{dd}}+\mathbf{y}_{\mathrm{dr}}+\mathbf{y}_{\mathrm{rd}}+\mathbf{y}_{\mathrm{rr}} \\
& =\mathbf{T}\left(\left(\mathbf{1}+\mathbf{k}^{\mathrm{dr}}+\mathbf{k}^{\mathrm{rd}}+\mathbf{k}^{\mathrm{rr}}\right) \odot \boldsymbol{\beta}\right)+\mathbf{n} \\
& =\mathbf{T}(\mathbf{k} \odot \boldsymbol{\beta})+\mathbf{n},
\end{aligned}
$$

where $\mathbf{k}=\mathbf{1}+\mathbf{k}^{\mathrm{dr}}+\mathbf{k}^{\mathrm{rd}}+\mathbf{k}^{\mathrm{rr}}$ and $\mathbf{1}^{\widetilde{M} \widetilde{N}}$ is an $\widetilde{M} \widetilde{N} \times 1$ one vector. 


\section{MIMO Radar Detector in Compound-Gaussian Clutter}

5.1. GLRT Detector Design. The problem of detecting with MIMO radar can be formulated in terms of the following binary hypotheses test:

$$
\begin{aligned}
& H_{0}: \quad \mathbf{y}_{\mathrm{mp}}=\mathbf{n}, \\
& H_{1}: \quad \mathbf{y}_{\mathrm{mp}}=\mathbf{T}(\mathbf{k} \odot \boldsymbol{\beta})+\mathbf{n} .
\end{aligned}
$$

Standard GLRT is the following decision rule:

$$
\frac{\max _{\boldsymbol{\beta}, \lambda_{1}, \ldots, \lambda_{L}} f\left(\mathbf{Y} \mid H_{1}, \lambda_{1}, \ldots, \lambda_{L}, \boldsymbol{\beta}\right)}{\max _{\lambda_{1}, \ldots, \lambda_{L}} f\left(\mathbf{Y} \mid H_{0}, \lambda_{1}, \ldots, \lambda_{L}\right)} \underset{H_{0}}{>} \gamma_{G}
$$

where $f\left(\mathbf{Y} \mid H_{1}, \lambda_{1}, \ldots, \lambda_{L}, \boldsymbol{\beta}\right)$ and $f\left(\mathbf{Y} \mid H_{1}, \lambda_{1}, \ldots, \lambda_{L}\right)$ denote the probability density functions (pdfs) of the data under $H_{1}$ and $H_{0}$, respectively. And the pdfs can be written, respectively, as

$$
f\left(\mathbf{Y} \mid H_{1}, \lambda_{1}, \ldots, \lambda_{L}, \boldsymbol{\beta}\right)=\frac{1}{\pi^{N L} \operatorname{det}\left(\mathbf{C}_{\mathbf{n}}\right)} \exp \{-\operatorname{tr}(\Im)\},
$$

where $\mathfrak{I}=\mathbf{C}_{\mathbf{n}}^{-1}(\mathbf{y}-\mathbf{T}(\mathbf{k} \odot \boldsymbol{\beta})) \times(\mathbf{y}-\mathbf{T}(\mathbf{K} \odot \boldsymbol{\beta}))^{\dagger}$, and

$$
f\left(\mathbf{Y} \mid H_{0}, \lambda_{1}, \ldots, \lambda_{L}\right)=\frac{1}{\pi^{N L} \operatorname{det}\left(\mathbf{C}_{\mathbf{n}}\right)} \exp \left\{-\operatorname{tr}\left(\mathbf{C}_{\mathbf{n}}^{-1} \mathbf{y} \mathbf{y}^{\dagger}\right)\right\},
$$

where $\operatorname{det}(\bullet)$ and $\operatorname{tr}$ denote the determinant and the trace of a matrix, respectively.

The log-likelihood function of (21) is

$$
\begin{aligned}
\ln f\left(\mathbf{Y} \mid H_{0}, \lambda_{1}, \ldots, \lambda_{L}\right)= & -N L \ln \pi-L \ln \operatorname{det}(\mathbf{C}) \\
& -N \sum_{\ell=1}^{L} \ln \lambda_{\ell} \\
& -\sum_{\ell=1}^{L} \frac{\mathbf{y}^{\dagger}\left(E_{\ell \ell} \otimes \mathbf{C}^{-1}\right) \mathbf{y}}{\lambda_{\ell}}
\end{aligned}
$$

where $E_{\ell \ell}$ denotes the elementary matrix with component $e(\ell, \ell)=1$ and zero for others. Then, it is easy to obtain the Maximum Likelihood (ML) estimator of $\lambda$ under $H_{0}$; that is

$$
\widehat{\lambda}_{\ell}=\frac{\mathbf{y}^{\dagger}\left(E_{\ell \ell} \otimes \mathbf{C}^{-1}\right) \mathbf{y}}{N} .
$$

According to [21], we rewrite the log-likelihood function of (20) as

$$
\begin{aligned}
\ln f & \left(\mathbf{Y} \mid H_{1}, \lambda_{1}, \ldots, \lambda_{L}, \boldsymbol{\beta}\right) \\
= & -N L \ln \pi-L \ln \operatorname{det}(\mathbf{C})-N \sum_{\ell=1}^{L} \ln \lambda_{\ell} \\
& -\sum_{\ell=1}^{L} \frac{(\mathbf{y}-\mathbf{T}(\mathbf{k} \odot \boldsymbol{\beta}))^{\dagger}\left(E_{\ell \ell} \otimes \mathbf{C}^{-1}\right)(y-\mathbf{T}(\mathbf{k} \odot \boldsymbol{\beta}))}{\lambda_{\ell}} .
\end{aligned}
$$

Thus, the ML estimator of $\left\{\lambda_{\ell}\right\}_{\ell=1}^{L}$ is

$$
\widehat{\lambda}_{\ell}=\frac{(\mathbf{y}-\mathbf{T}(\mathbf{k} \odot \boldsymbol{\beta}))^{\dagger}\left(E_{\ell \ell} \otimes \mathbf{C}^{-1}\right)(\mathbf{y}-\mathbf{T}(\mathbf{k} \odot \boldsymbol{\beta}))}{N} .
$$

The estimator $\beta$ is [23]

$$
\widehat{\boldsymbol{\beta}}=\left((\mathrm{Tk})^{\dagger} \mathrm{C}_{\mathbf{n}}^{-1}(\mathrm{Tk})\right)(\mathrm{Tk})^{\dagger} \mathrm{C}_{\mathbf{n}}^{-1} \mathbf{y}
$$

Substituting the estimator $\left\{\widehat{\lambda}_{\ell}\right\}_{\ell=1}^{L}, \widehat{\boldsymbol{\beta}}$ under $H_{1}$ and $H_{0}$ into (19)-(21), the final GLRT becomes

$$
\prod_{\ell=1}^{L} \frac{\mathbf{y}^{\dagger}\left(E_{\ell \ell} \otimes \mathbf{C}^{-1}\right) \mathbf{y}}{(\mathbf{y}-\mathbf{T}(\mathbf{k} \odot \widehat{\boldsymbol{\beta}}))^{\dagger}\left(E_{\ell \ell} \otimes \mathbf{C}^{-1}\right)(\mathbf{y}-\mathbf{T}(\mathbf{k} \odot \widehat{\boldsymbol{\beta}}))} \stackrel{\stackrel{H_{1}}{>}}{>} \gamma_{H_{0}} .
$$

5.2. GLRT-LQ Detector Design. We rewrite the detection problem as

$$
\begin{array}{lc}
H_{0}: & y_{m, n}=z_{m, n}, \\
H_{1}: & y_{m, n}=\rho_{M_{m} N_{n}} \beta(m, n) T_{m, n}+z_{m, n},
\end{array}
$$

where $\rho_{M_{m} N_{n}}=\left(1+\rho_{M_{m} N_{n}}^{(\mathrm{dr})}+\rho_{M_{m} N_{n}}^{(\mathrm{rd})}+\rho_{M_{m} N_{n}}^{(\mathrm{rr})}\right) ; \rho_{M_{m} N_{n}}^{(\mathrm{dr})}$, $\rho_{M_{m} N_{n}}^{(\mathrm{rd})}, \rho_{M_{m} N_{n}}^{(\mathrm{rr})}$ are the amplitudes of reflect coefficient, because the grazing angles are different; the reflect coefficient $\rho_{M_{m} N_{n}}^{(\mathrm{dr})} \neq \rho_{M_{m} N_{n}}^{(\mathrm{rd})} \neq \rho_{M_{m} N_{n}}^{(\mathrm{rr})}$.

As the transmit-receive subarrays are widely separated, the clutter returns can be considered to be independent; hence, the low-grazing angle likelihood ratio test (LRT) detector for MIMO radar in the compound-Gaussian clutter is given by

$$
\log \frac{p\left(y \mid H_{0}\right)}{p\left(y \mid H_{1}\right)} \underset{<}{\stackrel{H_{1}}{<}} \gamma_{G-L Q}
$$

If we assume that covariance matrix $\mathbf{C}_{c}$ is known and according to [24], $p\left(y \mid H_{0}\right)$ and $p\left(y \mid H_{1}\right)$ are replaced by their Bayesian estimates, and, asymptotically, the generalized likelihood ratio test-linear quadratic (GLRT-LQ), extended to the MIMO case, is given by

$\Lambda(Y)$

$$
\begin{aligned}
& =\frac{p\left(Y \mid H_{1}\right)}{p\left(Y \mid H_{0}\right)}=\frac{\prod_{m, n} p\left(y_{m, n} \mid H_{1}\right)}{\prod_{m, n} p\left(y_{m, n} \mid H_{0}\right)} \\
& =\prod_{m, n}\left[1 \times\left(1-\frac{\left|T_{m, n}^{\dagger} \mathbf{C}_{m, n}^{-1} y_{m, n}\right|^{2}}{\left(T_{m, n}^{\dagger} \mathbf{C}_{m, n}^{-1} T_{m, n}\right)\left(y_{m, n}^{\dagger} M_{m, n}^{-1} y_{m, n}\right)}\right)^{-1}\right] \\
& \stackrel{H_{1}}{>} \gamma_{G-L Q}, \\
& { }_{H_{0}}
\end{aligned}
$$

where $\mathbf{C}_{m, n}$ is the covariance matrix for the $m-n$ transmitreceive pair. 
According to [24], the probability of false alarm $P_{\mathrm{fa}}$ is given by

$$
\begin{aligned}
P_{\mathrm{fa}} & =P\left(\prod_{i=1}^{I} \Lambda_{i}\left(y_{i}\right)>\gamma_{G-L Q} \mid H_{0}\right) \\
& =\gamma_{\mathrm{G}-L \mathrm{Q}}^{-L+1} \sum_{i=0}^{I-1} \frac{(W-I)^{i}}{i !}\left(\ln \gamma_{\mathrm{G}-L \mathrm{Q}}\right)^{i},
\end{aligned}
$$

where $I=\widetilde{M} \widetilde{N}$ and $W=M_{m} N_{n}$.

The probability of detection $P_{d}$ is given by

$$
P_{d}=P\left(\prod_{i=1}^{I} \Lambda_{i}\left(y_{i}\right)>\gamma_{G-L Q} \mid H_{0}\right) .
$$

For a given signal-to-clutter ratio (SCR), denoted by $\mathrm{SCR}_{m, n}$, the amplitude of $\beta(m, n)$ is given by

$$
|\beta(m, n)|=\sqrt{\operatorname{SCR}_{m, n} \sigma^{2}},
$$

where $\sigma^{2}$ is the clutter power. In this paper, we consider that $|\beta(m, n)|$ is the same for all $m$ and $n$.

\section{Numerical Simulations}

This section is devoted to the performance assessment of the GLRT and GLRT-LQ detectors in low-grazing angle for MIMO radar, when the texture component of clutter distributed as gamma distribution, leading to the wellknown $K$ clutter model. Since the closed-form expressions of the GLRT detector for the probability of the detection and of alarm are not available, we resort to standard Monte Carlo.

In our first example, we, respectively, analyze the GlRTLQ detectors considering multipath effect and without considering multipath effect. Assume MIMO radar is with three transmit antennas and two receive antennas, the heights of transmit arrays are fixed at $100 \mathrm{~m}, 200 \mathrm{~m}$, and $300 \mathrm{~m}$, the height of receive arrays are fixed at $100 \mathrm{~m}$, and $200 \mathrm{~m}$, and the target's height is fixed at $200 \mathrm{~m}$. The $(p, q)$, the element of the covariance matrix of the speckle component, is chosen as

$$
\mathbf{C}_{p, q}=\sigma^{2} \times 0.9^{|p-q|} \times \exp \left[j\left(\frac{\pi}{2}\right)(p-q)\right] .
$$

Here, we select $\sigma^{2}=10.17, L=16$. We define the signal-toclutter pulse noise ratio (SCNR) by [25]

$$
\mathrm{SCNR}=\frac{1}{L} \frac{\sum_{\ell=1}^{L}[\mathbf{T}(\mathbf{k} \odot \boldsymbol{\beta})]^{\dagger}[\mathbf{T}(\mathbf{k} \odot \boldsymbol{\beta})]}{E\{u\} \operatorname{tr}\left\{C_{n}\right\}} .
$$

Figure 3 depicts the detection performance using GLRTLQ detectors, as a function of the SCNR. The probability of false alarm is fixed at $P_{\mathrm{fa}}=10^{-4}$. For the given SCNR, the detection performance with multipath outperforms the one without considering multipath effect.

Figure 4 depicts the detection performance using GLRT detector, as a function of the SCNR. For the given SCNR, the

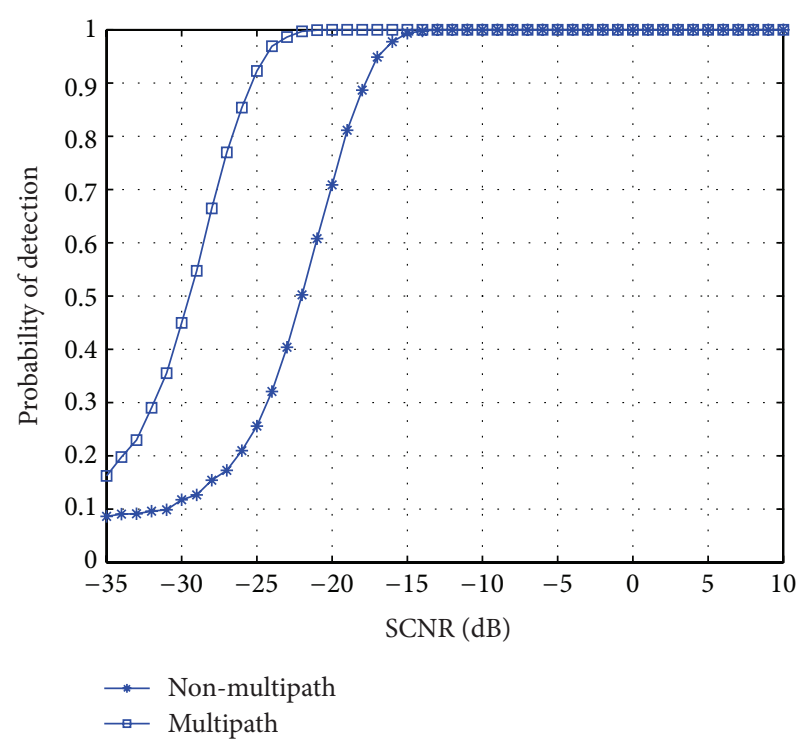

FIGURE 3: GLRT-LQ detector performance in low-grazing angle.

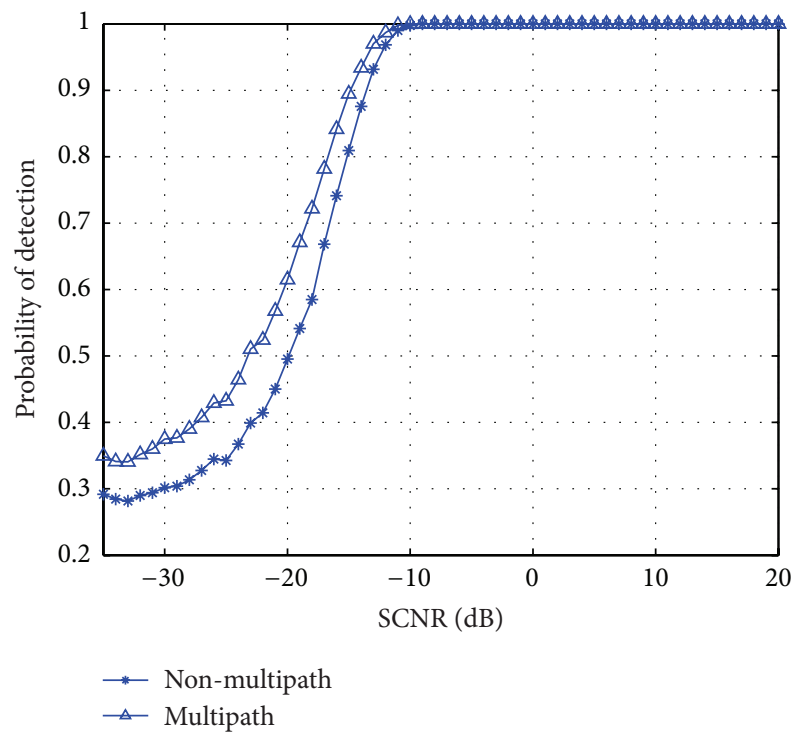

FIGURE 4: GLRT detector performance in low-grazing angle.

detection performance with multipath outperforms the one without considering multipath effect.

Figure 5 depicts the performance comparison between GLRT-LQ and GLRT detectors. From Figure 5, GLRT-LQ detector outperforms the GLRT detector in low-grazing angle for MIMO radar, respectively, with and without considering multipath effects.

Figures 6 and 7 depict the detection performance of GLRT-LQ and GLRT detector with different antenna numbers, respectively, as a function of SCNR. The probability of false alarm is set at $P_{\mathrm{fa}}=10^{-4}$; the transmit antenna and receive antenna are set at 2,4 , and 6 , respectively. Figures 6 and 7 show that GLRT-LQ or GLRT detector can obtain 


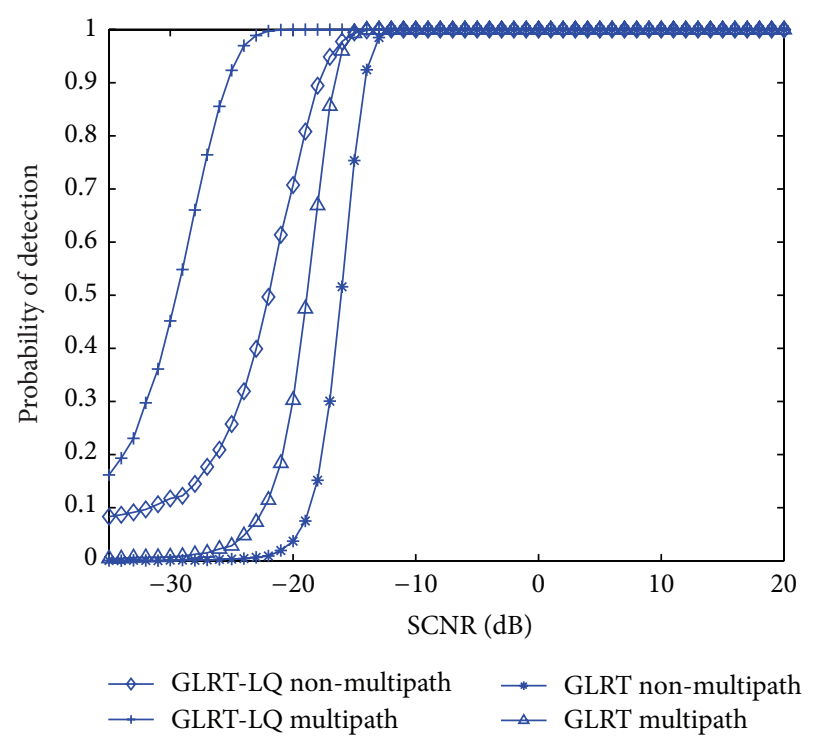

FIGURE 5: Comparison of GLRT-LQ detector and GLRT detector.

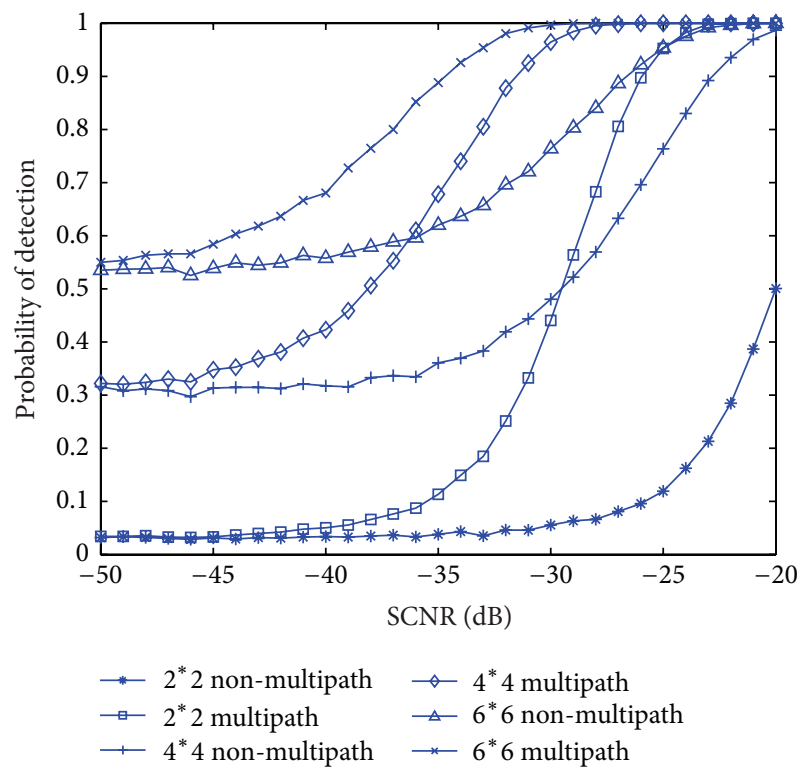

FIGURE 6: Detection performance of GLRT-LQ detector with different numbers.

better detection performance when there are more number of transmit antennas and receive antennas.

Figures 8 and 9 depict the detection performance of GLRT-LQ and GLRT detector with target height, respectively; the heights of target are fixed at $200 \mathrm{~m}, 400 \mathrm{~m}$, and $600 \mathrm{~m}$ and the probabilities of false alarm are fixed at $P_{\mathrm{fa}}=$ $10^{-4}$. Figures 8 and 9 show that the detection performance varies with the height of target. We can see that the performance increases with the height of target under the lowgrazing scene. However, the performance does not always increase with the height of target, just as Figure 10. When the height of target is $1600 \mathrm{~m}$, the condition of low-grazing angle

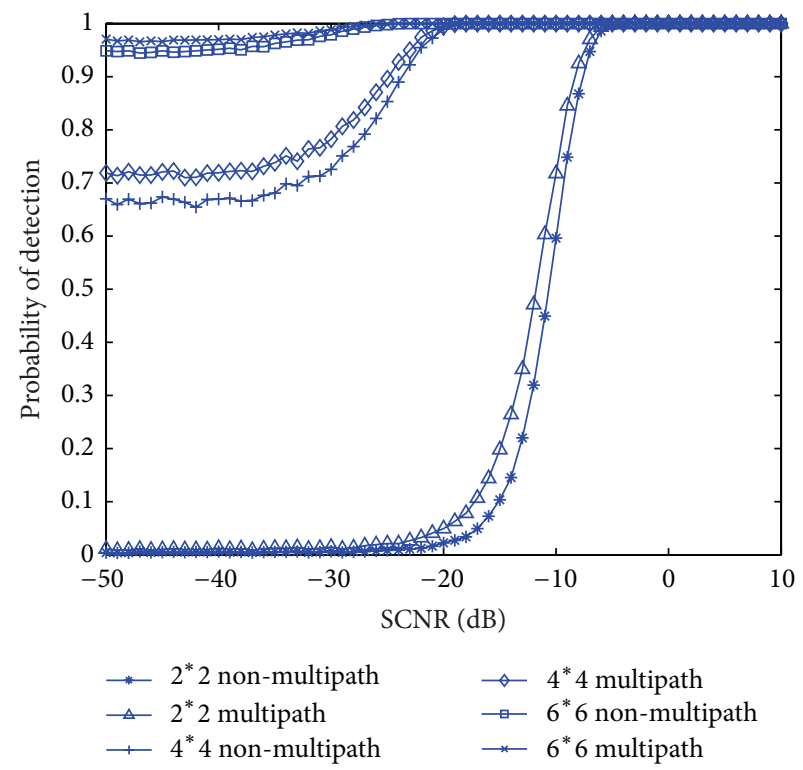

FIGURE 7: Detection performance of GLRT detector with different numbers.

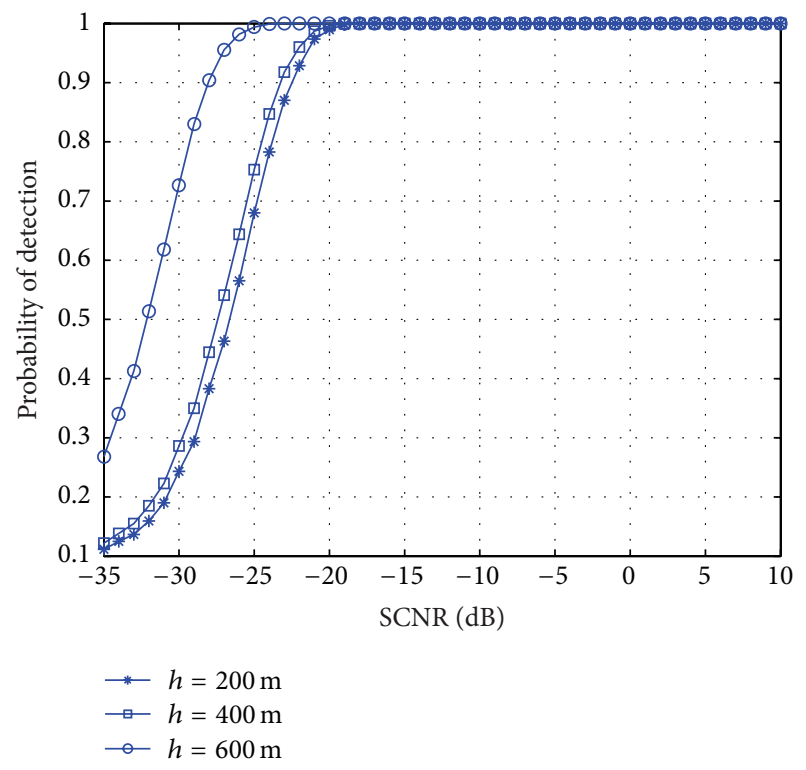

FIGURE 8: GLRT-LQ detector detection performance varies with the height of target.

is not satisfied. If we still take it for low-grazing angle, the detection performance will decrease.

\section{Conclusion}

In this paper, we have introduced the concept of reflection coefficient under considering curved earth effect and introduced general signal model for MIMO radar in low-grazing angle, firstly. Then, we have derived the GLRT-LQ and GLRT detectors, respectively. Furthermore, we have compared the performance of GLRT-LQ and GLRT detector for MIMO 


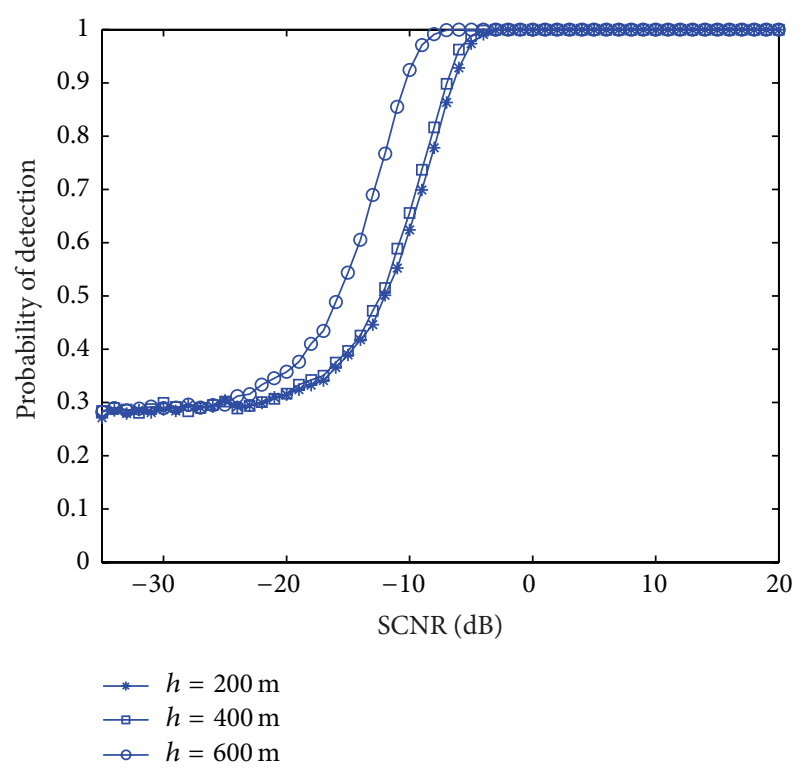

FIGURE 9: GLRT detector detection performance varies with the height of target.

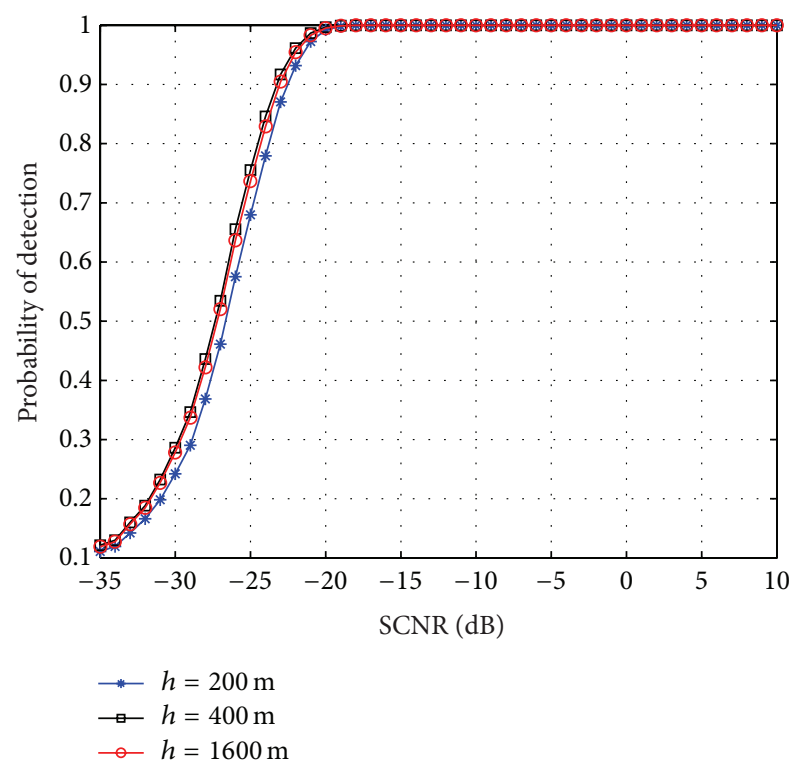

FIGURE 10: GLRT-LQ detector detection performance varies with the height of target.

radar between with multipath and without multipath effects. The simulation results have shown the importance of multipath effects for target detection in low-grazing angle and demonstrated that GLRT-LQ detector outperforms the GLRT detector in low-grazing angle.

\section{Acknowledgments}

The authors wish to thank the anonymous reviewers for their efforts in providing comments that have helped to significantly enhance the quality of this paper. This work was supported in part by the National Science Foundation of China under Grant no. 61302142.

\section{References}

[1] J. Li and P. Stoica, "MIMO radar with colocated antennas: review of some recent work," IEEE Signal Processing Magazine, vol. 24, no. 5, pp. 106-114, 2007.

[2] A. M. Haimovich, R. S. Blum, and L. J. Cimini, "MIMO radar with widely separated antennas," IEEE Signal Processing Magazine, vol. 25, no. 1, pp. 116-129, 2008.

[3] I. Bekkerman and J. Tabrikian, "Target detection and localization using MIMO radars and sonars," IEEE Transactions on Signal Processing, vol. 54, no. 10, pp. 3873-3883, 2006.

[4] K. W. Forsythe, D. W. Bliss, and G. S. Fawcett, "Multipleinput multiple-output (MIMO) radar: performance issues," in Proceedings of the 38th Asilomar Conference on Signals, Systems and Computers, pp. 310-315, Pacific Grove, Calif, USA, November 2004.

[5] P. Stoica, J. Li, and Y. Xie, "On probing signal design for MIMO radar," IEEE Transactions on Signal Processing, vol. 55, no. 8, pp. 4151-4161, 2007.

[6] J. Li and P. Stoica, MIMO Radar Signal Processing, Wiley-IEEE Press, New York, NY, USA, 2008.

[7] Q. He, R. S. Blum, H. Godrich, and A. M. Haimovich, "Target velocity estimation and antenna placement for MIMO radar with widely separated antennas," IEEE Journal on Selected Topics in Signal Processing, vol. 4, no. 1, pp. 79-100, 2010.

[8] H. W. Chen, Y. P. Chen, X. Li, and Z. W. Zhuang, "Extended ambiguity function for bistatic MIMO radar," Journal of Systems Engineering and Electronics, vol. 23, no. 2, pp. 109-114, 2012.

[9] N. H. Lehmann, A. M. Haimovich, R. S. Blum, and L. Cimini, "High resolution capabilities of MIMO radar," in Proceedings of the 40th Asilomar Conference on Signals, Systems, and Computers (ACSSC '06), pp. 25-30, Pacific Groove, Calif, USA, November 2006.

[10] H. Godrich, V. M. Chiriac, A. M. Haimovich, and R. S. Blum, "Target tracking in MIMO radar systems: techniques and performance analysis," in Proceedings of the IEEE International Radar Conference (RADAR '10), pp. 1111-1116, May 2010.

[11] J. Guan and Y. Huang, "Detection performance analysis for MIMO radar with distributed apertures in Gaussian colored noise," Science in China F, vol. 52, no. 9, pp. 1688-1696, 2009.

[12] J. Tang, Y. Wu, Y. N. Peng, and X. T. Wang, "On detection performance and system configuration of MIMO radar," Science in China F, vol. 52, no. 7, pp. 1250-1257, 2009.

[13] J. Tang, Y. Wu, Y. Peng, and X. Wang, "On detection performance of MIMO radar for Rician target," Science in China F, vol. 52, no. 8, pp. 1456-1465, 2009.

[14] E. Fishler, A. Haimovich, R. S. Blum, L. J. Cimini Jr., D. Chizhik, and R. A. Valenzuela, "Spatial diversity in radars: models and detection performance," IEEE Transactions on Signal Processing, vol. 54, no. 3, pp. 823-838, 2006.

[15] T. Aittomaki and V. Koivunen, "Performance of MIMO radar with angular diversity under swerling scattering models," IEEE Journal on Selected Topics in Signal Processing, vol. 4, no. 1, pp. 101-114, 2010.

[16] Y. Jin, J. M. F. Moura, and N. O’Donoughue, “Time reversal in multiple-input multiple-output radar," IEEE Journal on Selected Topics in Signal Processing, vol. 4, no. 1, pp. 210-225, 2010. 
[17] D. K. Barton, "Low angle tracking," Proceedings of the IEEE, vol. 62, no. 6, pp. 687-704, 1974.

[18] S. L. Silon and B. D. Carlson, "Radar detection in multipath," IEE Proceedings F, vol. 146, no. 1, pp. 45-54, 1999.

[19] T. Lo and J. Litva, "Use of a highly deterministic multipath signal model in low-angle tracking," IEE Proceedings F, vol. 138, no. 2, pp. 163-171, 1991.

[20] M. I. Skolnik, Radar Handbook, McGraw-Hill, New York, NY, USA, 2nd edition, 1990.

[21] M. A. Richards, Fundamentals of Radar Signal Processing, McGraw-Hill, New York, NY, USA, 2005.

[22] K. D. Ward, C. J. Baker, and S. Watts, "Maritime surveillance radar. Part 1: radar scattering from the ocean surface," IEE proceedings $F$, vol. 137, no. 2, pp. 51-62, 1990.

[23] F. Gini, A. Farina, and G. Foglia, "Effects of foliage on the formation of K-distributed SAR imagery," Signal Processing, vol. 75, no. 2, pp. 161-171, 1999.

[24] G. Cui, L. Kong, and X. Yang, "Multiple-input multiple-output radar detectors design in non-Gaussian clutter," IET Radar, Sonar and Navigation, vol. 4, no. 5, pp. 724-732, 2010.

[25] M. Akcakaya and A. Nehorai, "Adaptive MIMO radar design and detection in compound-gaussian clutter," IEEE Transactions on Aerospace and Electronic Systems, vol. 47, no. 3, pp. 2200-2207, 2011. 

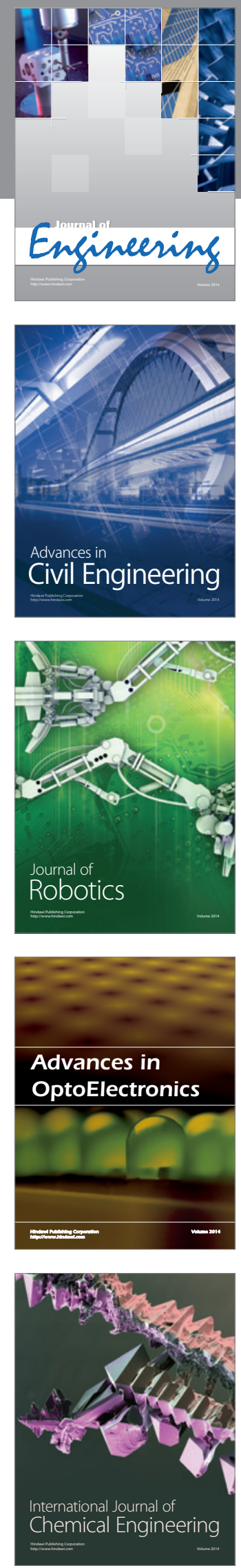

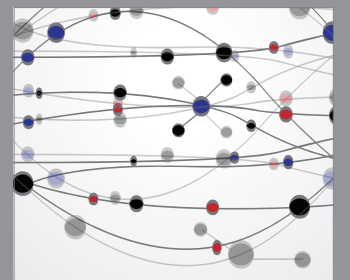

The Scientific World Journal
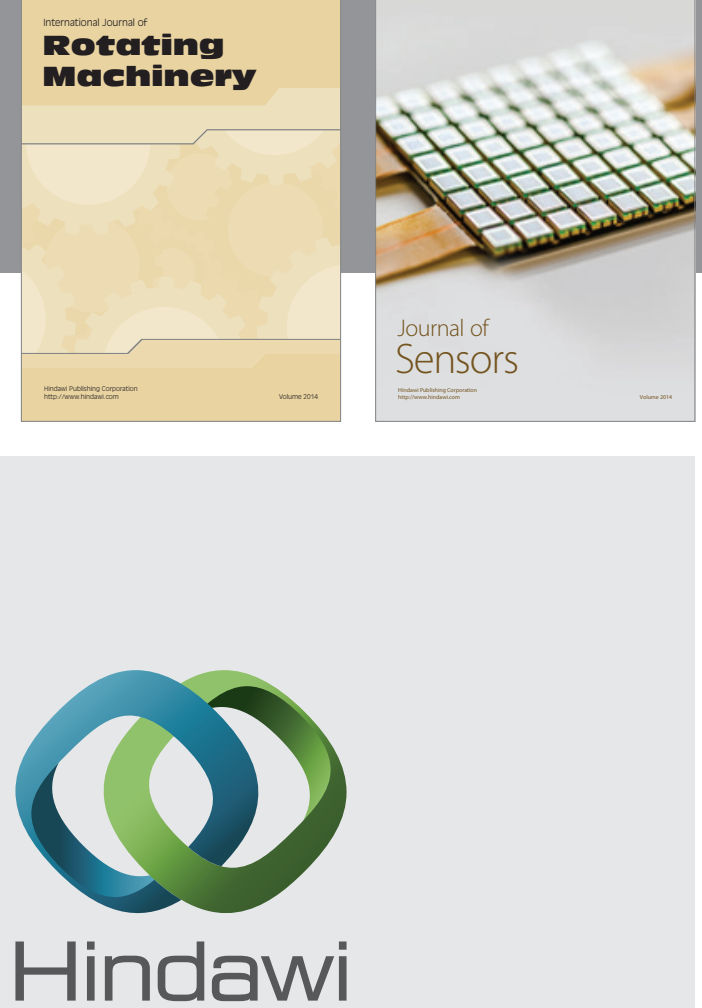

Submit your manuscripts at http://www.hindawi.com
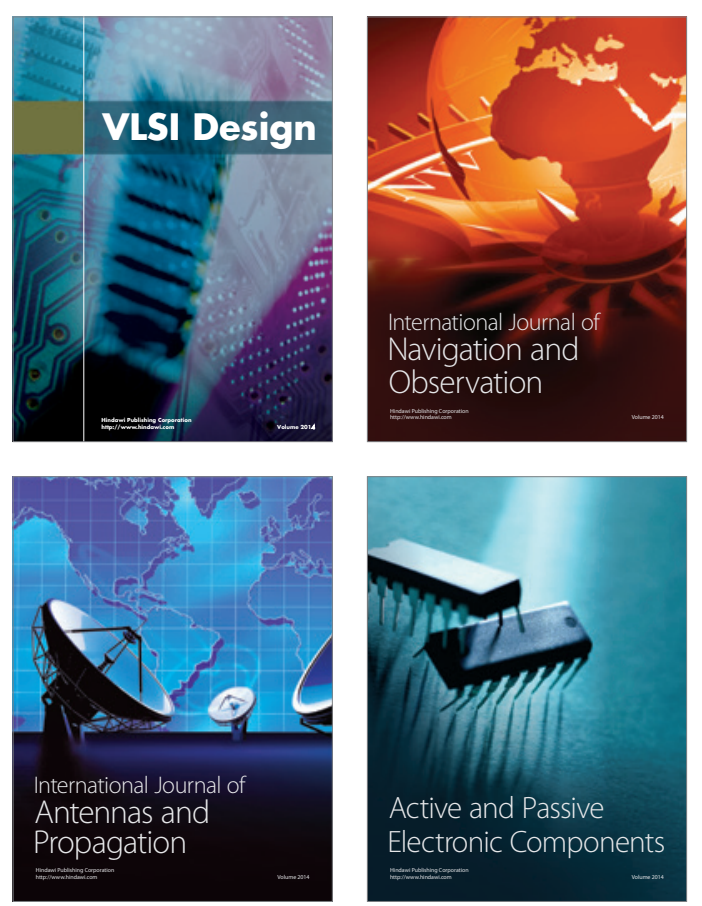
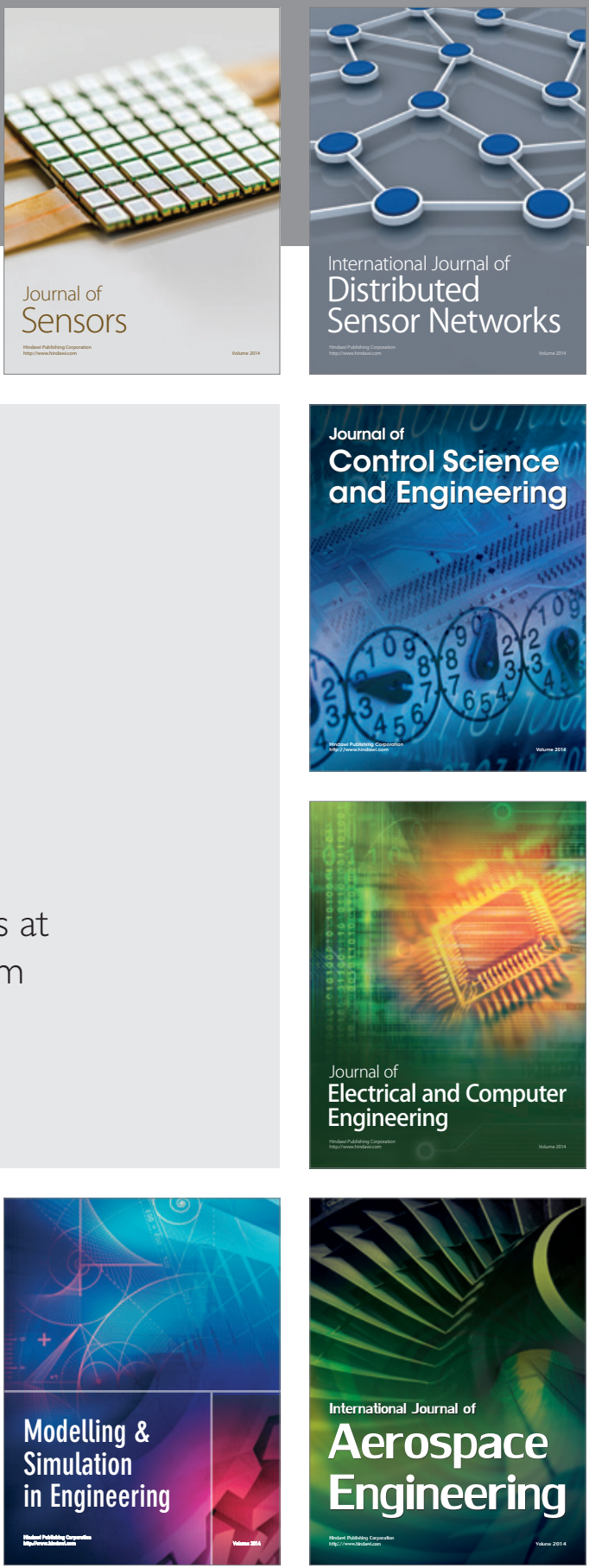

Journal of

Control Science

and Engineering
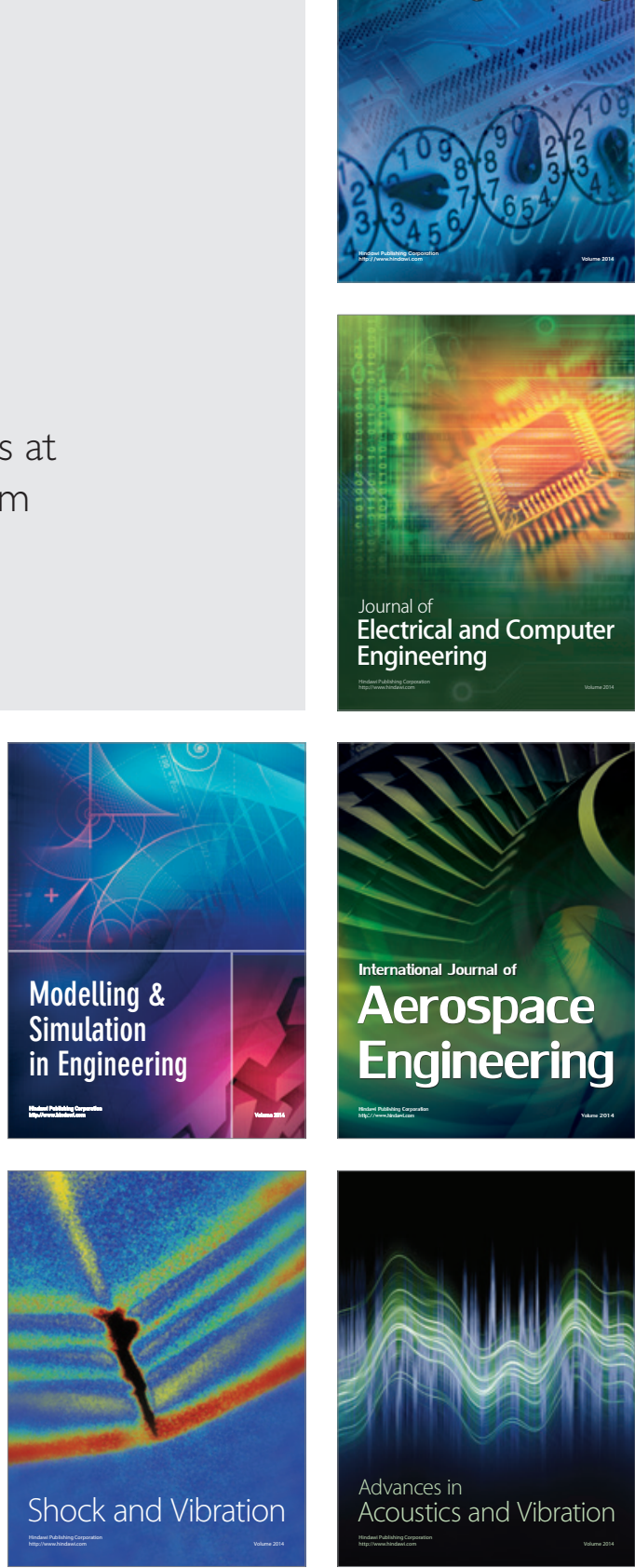